\title{
Fine motor skills in South African children with symptoms of ADHD: influence of subtype, gender, age, and hand dominance Anneke Meyer*1,3 and Terje Sagvolden ${ }^{2,3}$
}

Address: ${ }^{1}$ School of Health Sciences, University of Limpopo, Private Bag X1106, 0727 Sovenga, South Africa, ${ }^{2}$ Department of Physiology, Institute of Basic Medical Sciences, University of Oslo, NO-0317 Oslo, Norway and ${ }^{3}$ Centre for Advanced Study at the Norwegian Academy for Science and Letters, NO-0271 Oslo, Norway

Email: Anneke Meyer* - jad1@mweb.co.za; Terje Sagvolden - terje@sagvolden.com

* Corresponding author

Published: 09 October 2006

Behavioral and Brain Functions 2006, 2:33 doi:10.1 186/1744-908I-2-33
Received: 22 June 2005

Accepted: 09 October 2006

This article is available from: http://www.behavioralandbrainfunctions.com/content/2/1/33

(C) 2006 Meyer and Sagvolden; licensee BioMed Central Ltd.

This is an Open Access article distributed under the terms of the Creative Commons Attribution License (http://creativecommons.org/licenses/by/2.0), which permits unrestricted use, distribution, and reproduction in any medium, provided the original work is properly cited.

\begin{abstract}
Background: Motor problems, often characterised as clumsiness or poor motor coordination, have been associated with ADHD in addition to the main symptom groups of inattention, impulsiveness, and overactivity. The problems addressed in this study were: (I) Are motor problems associated with ADHD symptoms, also in African cultures? (2) Are there differences in motor skills among the subtypes with ADHD symptoms? (3) Are there gender differences? (4) Is there an effect of age? (5) Are there differences in performance between the dominant and nondominant hand?
\end{abstract}

Method: A total of 528 children ( 264 classified as having symptoms of ADHD and 264 matched comparisons) of both genders and from seven different South African ethnic groups participated in the study. They were assessed with three simple, easy to administer instruments which measure various functions of motor speed and eye-hand coordination: The Grooved Pegboard, the Maze Coordination Task, and the Finger Tapping Test. The results were analysed as a function of subtype, gender, age, and hand dominance.

Results: The findings indicate that children with symptoms of ADHD performed significantly poorer on the Grooved Pegboard and Motor Coordination Task, but not on the Finger Tapping Test than their comparisons without ADHD symptoms. The impairment was most severe for the subtype with symptoms of ADHD-C (combined) and less severe for the subtypes with symptoms of ADHD-PI (predominantly inattentive) and ADHD-HI (predominantly hyperactive/impulsive). With few exceptions, both genders were equally affected while there were only slight differences in performance between the dominant and non-dominant hand. The deficiencies in motor control were mainly confined to the younger age group $(6-9 \mathrm{yr})$.

Conclusion: An association between the symptoms of ADHD and motor problems was demonstrated in terms of accuracy and speed in fairly complex tasks, but not in simple motor tests of speed. This deficiency is found mainly in the children with ADHD-C symptoms, but also to a lesser degree in the children with symptoms of ADHD-PI and ADHD-HI. 


\section{Background}

Attention-Deficit/Hyperactivity Disorder (ADHD) is a prevalent, heterogeneous, and debilitating neurodevelopmental disorder with an early onset. The disorder consists of a persistent pattern of inattentiveness, impulsiveness, and/or hyperactivity that is inconsistent with the child's developmental level [1]. ADHD is the most common child psychiatric disorder in Europe and the United States, affecting 3-10\% of primary school children [2-4]. The disorder is generally more prevalent in males, but more severe in females [5]. Male-to-female ratios vary from 9:1 to $6: 1$ in clinic-referred samples, but is only approximately 3:1 in population-based ones [3]. ADHD is associated with proneness for repeated accidents, depressive and anxiety disorders, learning disabilities, and school failure [4,6-9]. The disorder is also associated with later increased incidence of substance abuse if not diagnosed and treated at an early age [10]. Thus, the economic, educational, social, and personal costs of ADHD are enormous.

Although referral practices and assessment procedures are neither well developed nor standardized in developing countries [11], ADHD is as prevalent and has the same sex ratios on the African continent as in Western countries [12-14] suggesting that ADHD is caused by the same fundamental neurobiological factors probably hypofunctioning dopamine systems influencing among other functions learning and behavioural planning $[15,16]$.

DSM-IV [1] identifies three subtypes of the disorder, namely: ADHD predominantly inattentive type (ADHDPI) if six (or more) symptoms of inattention (but fewer than six symptoms of hyperactivity-impulsiveness) have persisted for at least 6 months; ADHD predominantly Hyperactive-Impulsive Type (ADHD-HI), if six (or more) symptoms of hyperactivity-impulsiveness (but fewer than six symptoms of inattention) have persisted for at least 6 months; and ADHD combined type (ADHD-C), if at least six symptoms of inattention and at least six symptoms of hyperactivity-impulsiveness have persisted for at least 6 months.

Besides the symptoms of inattention, hyperactivity, and impulsiveness, the ADHD child's motor ability is frequently significantly lower than would be expected of his age and level of intellectual functioning [1]. The wide range of motor problems include delays in achieving motor milestones, problems with movement planning and execution (reaction time, movement time, accuracy, and variability) [17], and parameter setting (not to be able to adapt their task performance to environmental requirements) [18]. Children with ADHD who experience motor problems often display deficits in requiring complex coordinations of movement, such as handwriting $[2,18]$.
These problems may interfere with the ADHD child's daily functioning and influence their academic performance [19].

Development Coordination Disorder (DCD) is a disorder with marked impairment in the development of motor coordination which cannot be attributed to a general medical condition or mental retardation [1]. Although DSM-IV [1] does not link DCD with ADHD, the disorders co-occur in $\sim 50 \%$ of cases $[18,20,21]$ through a shared, additive genetic component between most subtypes of ADHD and DCD [22]. The children diagnosed with DCD who have problems with fine motor skills, are usually strongly linked to the ADHD-PI subtype [23]. In the Scandinavian countries the term DAMP (deficits in attention, motor control, and perception) is sometimes used for children with ADHD+DCD [8,24,25]. DAMP has been defined as a neurodevelopmental dysfunction syndrome with a high degree of comorbidity. Motor clumsiness is however, also associated with a variety of developmental disorders: learning disability [26], reading disorder [27], oppositional defiance disorder (ODD) [28] autism [17] and Asperger's syndrome [29,30].

The aspects of motor performance to be assessed in the present study were manual dexterity, complex coordination, and motor planning (by the Grooved Pegboard and the Maze Coordination Task), and manual dexterity (by the Finger Tapping Task).

The problems addressed were: Are motor problems associated with ADHD, also in African cultures? Are there differences in motor skills among the ADHD subtypes? Are there gender differences? Is there an effect of age? Are there differences in performance between the dominant and non-dominant hand?

\section{Method \\ Sample}

Children in the Limpopo Province of South Africa, consisting of 7 ethnic groups (Northern Sotho, Venda, Tsonga, Tswana, North Ndebele, Bolobedu, and Afrikaans) were recruited from a school-based population. The 528 children ( 378 boys and 150 girls) were recruited following screening for ADHD of the general population of primary school children representative of all socio-economic levels. Written permission was obtained from the Department of Education, Limpopo Province, as well of the principals of the selected schools.

The Disruptive Behavior Disorders (DBD) rating scale $[31,32]$ was standardized for the populations of the Limpopo province of South Africa in an earlier study [14] and used as the screening instrument. Participation was voluntary. Informed consent was obtained from the child's par- 
Table I: Sample characteristics

\begin{tabular}{|c|c|c|c|c|c|c|c|c|}
\hline Subtype & Afrikaans & $\begin{array}{l}\text { Northern } \\
\text { Sotho }\end{array}$ & Tsonga & Venda & $\begin{array}{c}\text { North } \\
\text { Ndebele }\end{array}$ & Tswana & Bolobedu & Total \\
\hline \multicolumn{9}{|l|}{ ADHD-HI } \\
\hline \multicolumn{9}{|l|}{ Boys } \\
\hline $6-9 \mathrm{yr}$ & 6 & 5 & 1 & 2 & 1 & 3 & 5 & 23 \\
\hline $10-13 \mathrm{yr}$ & 3 & 2 & 0 & 4 & 4 & 9 & 6 & 30 \\
\hline \multicolumn{9}{|l|}{ Girls } \\
\hline $6-9 \mathrm{yr}$ & 1 & 2 & 3 & 4 & 1 & 1 & 1 & 13 \\
\hline $10-13 \mathrm{yr}$ & 2 & 1 & 2 & 0 & 1 & 0 & 2 & 8 \\
\hline \multicolumn{9}{|l|}{ ADHD-PI } \\
\hline \multicolumn{9}{|l|}{ Boys } \\
\hline $6-9 \mathrm{yr}$ & 4 & I & 5 & 10 & 6 & 3 & 4 & 33 \\
\hline $10-13 \mathrm{yr}$ & 4 & 3 & 3 & 5 & 3 & 2 & 8 & 28 \\
\hline \multicolumn{9}{|l|}{ Girls } \\
\hline $6-9 \mathrm{yr}$ & 1 & 4 & 3 & 1 & 1 & I & 2 & 13 \\
\hline $10-13 \mathrm{yr}$ & 1 & 2 & 5 & 3 & 2 & 4 & 3 & 20 \\
\hline \multicolumn{9}{|l|}{ ADHD-C } \\
\hline \multicolumn{9}{|l|}{ Boys } \\
\hline $6-9 \mathrm{yr}$ & 3 & 7 & 8 & 5 & 4 & 4 & 6 & 37 \\
\hline $10-13 \mathrm{yr}$ & 4 & 6 & 7 & 6 & 4 & 6 & 5 & 38 \\
\hline \multicolumn{9}{|l|}{ Girls } \\
\hline $6-9 \mathrm{yr}$ & 3 & 2 & 1 & 2 & 2 & I & 2 & 13 \\
\hline $10-13 \mathrm{yr}$ & 0 & 4 & 1 & 0 & 0 & 2 & 1 & 8 \\
\hline \multicolumn{9}{|l|}{ Non-ADHD } \\
\hline \multicolumn{9}{|l|}{ Boys } \\
\hline $6-9 \mathrm{yr}$ & 13 & 13 & 14 & 17 & 11 & 10 & 15 & 93 \\
\hline $10-13 \mathrm{yr}$ & 13 & 11 & 10 & 15 & 11 & 17 & 19 & 96 \\
\hline \multicolumn{9}{|l|}{ Girls } \\
\hline $6-9 \mathrm{yr}$ & 5 & 8 & 7 & 7 & 4 & 3 & 4 & 38 \\
\hline $10-13 \mathrm{yr}$ & 3 & 7 & 8 & 3 & 3 & 6 & 7 & 37 \\
\hline Total & 68 & 78 & 78 & 84 & 58 & 72 & 90 & 528 \\
\hline
\end{tabular}

ents or guardians. Both teachers and parents were given the rating scale to complete. Only the teacher's ratings were used for the screening, since the return of the parent's rating scale was below 50\%, probably because many children either did not live with their parents or the parents were illiterate. Teacher ratings are usually regarded as an accurate measure of assessment [6]. The return of the teacher's rating scale was however close to $100 \%$. The children meeting the criteria for inclusion into the groups with ADHD symptoms ( $7 \%$ ) were selected for further testing. They were matched for gender, age, and ethnic group with children who did not meet the inclusion criteria, obtained from the screening process.

Children were divided into a group with symptoms of ADHD and a comparison group without ADHD symptoms (Table 1), based on teacher ratings on the DBD rating scale [31,32]. Cut off points for the group with ADHD symptoms ( $95^{\text {th }}$ percentile or above) and comparison group
( $85^{\text {th }}$ percentile or below) were based on the results from the prevalence study [14] in which more than 6000 children in the Limpopo Province were rated on the DBD. According to the norms, scores on hyperactive/impulsive related items less than 15 and inattentive items less than 17 were regarded as comparisons. Scores higher than 18 on the Hyperactive/Impulsive items were classified as having symptoms of $A D H D-H I$ and higher than 21 on the Inattention items were classified as having ADHD-PI symptoms. If the criteria were met on both types of items they were classified as having symptoms of ADHD-C.

The final sample consisted of children from seven ethnic groups inhabiting the Limpopo Province (Table 1). Children with an IQ lower than 80 and/or with a history of neurological problems (e.g. epilepsy, head injuries, cerebral palsy, or cerebral malaria) were excluded. None of the children was on psychostimulant medication at the time of testing. 


\section{Instruments}

Assessment of, and research, on ADHD in developing countries like South Africa could be improved with standardized tests reliably differentiating between children with and without ADHD symptoms. The tests selected in the present study measure various aspects of fine motor functions, mainly assess basic non-verbal skills. They are simple, inexpensive, easy to transport to and use in remote rural areas without the conveniences of Western settings.

The three tests which measure different aspects of fine manual motor skills were the Grooved Pegboard Test [33] (distal, complex fine motor coordination and psychomotor speed), the Maze Coordination Task [33] (tactual motor coordination skills) and the Finger Tapping Test [34] (pure motor speed).

The Grooved Pegboard apparatus (Lafayette Instrument Company, \#4202) consists of a metal board $(10 \times 10 \mathrm{~cm})$ that contains a $5 \times 5$ set of holes each with a groove oriented randomly in different directions. Twenty-five round metal pegs with a ridge running lengthwise have to be rotated into the correct position for insertion into the holes. The child is instructed to insert the pegs as fast as possible, completing one row before starting on the next. The test is performed once with each hand, always starting with the dominant hand. Time to completion (in s) is the final score for each hand.

The following instruction is given in the child's own language:

"You are now going to put each of these pegs into the holes of this board (show). You can only use one hand. Pick up one peg at a time. Notice that the pegs are not round, neither are the holes in the board. In order to insert it you will have to rotate the peg so that it fits exactly (show two pegs, let the child try the three next, then remove all five pegs from the holes). When I tell you to start, you shall start over here (point to the upper left hole if the child is using its right hand and to the upper right hole if the child is using its left hand), fill this upper row, continue on the next, and so on until all the pegs are inserted. Try to be quick. Use only your (dominant/non-dominant) hand."

The Maze Coordination Task (Lafayette Instrument Company, \#2706A) is a simple maze without blind alleys. The maze is placed at 60 degree angle with the table. The child is required to go through the maze with an electric stylus, trying not to touch the sides. The stylus is connected to an electronic clock and a counter, which record the number of contacts the stylus is making with the sides (counter) and the cumulative time these contacts last (timer). The aim is to move the stylus through the maze, without touching the sides. There is no speed require- ment. The test is performed twice with each hand. The total sum of touches and cumulative time of contact of two trials with the same-side hand are the final scores.

The following instruction is given in the child's own language:

"In this test, take this stylus and move it through the maze all the way to here (point). Try to avoid touching the sides (show). Do this with about this speed. (Show by moving stylus through about 1/4 of the maze). You do not have to rush, if you move too quickly you will make more errors. Try to be accurate. Start with your (dominant) hand. Do not rest your hand or arm against anything".

The Finger Tapping Test apparatus (Marquardt, type 0925.0201) is a micro-switch operated by a key consisting of a metal arm and a round disk (20 $\mathrm{mm}$ in diameter). The key is placed at the short end of a $223 \mathrm{~mm} \times 151 \mathrm{~mm} \times 38$ $\mathrm{mm}(\mathrm{h})$ plastic box where the operating hand is to be rested. The length of the metal arm from the micro-switch to the centre of the disc is $60 \mathrm{~mm}$. The switch needs $\sim 65 \mathrm{~g}$ dead weight to close. An electronic counter records the number of micro-switch closings (taps). The child has to press the switch $\sim 15 \mathrm{~mm}$ to activate the counter. It is important that the hand is rested in a constant position in contact with the surface of the plastic box to ensure that only the index finger is moving. A stopwatch is used to time each 10-s trial. The child may rest at any time between trials, but is told to take a break at least after every third trial. For each hand, the test is terminated after ten trials, or when five consecutive trials do not vary by more than five taps. The means of the five trials with the highest number of taps are computed for each hand and used as the final scores.

The following instruction is given in the child's own language:

"Can you, please, show me how fast you can press this button with your (dominant) index finger? (If the child is small, touch the index finger. Demonstrate what the child has to do). Rest your arm in a comfortable position and try for yourself. You have to press the button all the way down and release it, or the counter will not work properly. Keep your wrist and arm still and remember to press as fast as you can. I will tell you when to start and when to stop."

\section{Procedure}

The children were always tested by a tester fluent in the child's own language. Most assessments of motor functions of the South African children were done at their schools during school hours. The exceptions were the children whose school was within a radius of $2 \mathrm{~km}$ from the 
University and the children referred for assessment, which were tested at the University Clinic.

To assess hand dominance, the children were asked to hit a nail with a small hammer, throw a ball, and to write their name. Experimental tests were presented in the following order: Grooved Pegboard, Maze Coordination Task, and Finger Tapping Test. For the Afrikaans group, the IQ was established with the Senior South African Individual Scale (SSAIS) [35]. As there are no standardized IQ tests for the indigenous African populations, Raven's progressive matrices was used to estimate IQ $[36,37]$. This test is considered to be culture-fair [38]. The actual testing procedure for each child lasted about $45 \mathrm{~min}$ and was conducted by intern clinical psychologists.

\section{Statistical analysis}

Raw scores were converted to standard scores (z-scores) for each ethnic group, to eliminate the effects of possible differences between testers and the translation of instructions. Group differences on demographic variables were analysed using analysis of variance (ANOVA) using the Statistica 6.1 programme [39]. The results were analysed twice with $4 \times 2 \times 2$ (subtype $\times$ gender $\times$ hand dominance and subtype $\times$ age group $\times$ hand dominance) ANOVA's for independent samples, with dominant vs. non-dominant hand as within-child repeated measure. Post-hoc tests consisted of multiple comparisons using the Bonferroni correction.

\section{Results}

\section{Analysis I: gender effects}

In general, children with high scores on the Disruptive Behavior Disorders (DBD) rating scales, who were classified as having ADHD symptoms, performed more poorly on the Grooved Pegboard and Motor Coordination Task than the comparison group without ADHD symptoms. Further, girls performed worse than boys. There were no differences in performance on the Finger Tapping Test.

Figures $1 \mathrm{~A}, 2 \mathrm{~A}$ and $3 \mathrm{~A}$ present the performances for each of the three tasks for both hands for the subtypes with ADHD symptoms and the non-ADHD comparison group for both genders. Figure 1A shows that the children with ADHD symptoms took more time than the comparisons to finish the task. This was the case for both the dominant and the non-dominant hand. Generally, the girls took longer to complete the task than the boys. This was however not the case for the group with Predominantly Inattentive symptoms (ADHD-PI). Figure 2A illustrates that the children with ADHD symptoms touched the side of the maze more often than the comparison group. The girls had overall more touches than the boys. This was the case with both hands. Figure 3A shows that there were no obvious differences in performance between the children with symptoms of the ADHD subtypes and the non-ADHD comparisons. There is also no obvious difference in performance between the genders.

The statistical results are shown in Table 2. For the Grooved Pegboard and the Maze Coordination Task there were statistically significant main effects of ADHD subtype and gender ( $\mathrm{ps}<0.001)$. On all three tests there was a statistically significant main effect of dominance (ps < $0.001)$.

Post-hoc testing (with Bonferroni correction) of the Grooved Pegboard and the Maze Coordination Task showed that in both tests, the children with symptoms of the ADHD-C subtype had the poorest performance. The differences, when compared with the non-ADHD group, were statistically significant for both genders. Both girls and boys with symptoms of the ADHD-PI subtype performed significantly poorer than the comparison group only in the Maze Coordination Task with the dominant hand. This was the case for both genders. When girls with ADHD-HI symptoms used their dominant hand, they performed significantly worse than the boys on the Grooved Pegboard as well as the Maze Coordination Task.

\section{Analysis 2: age effects}

Figures $1 \mathrm{~B}, 2 \mathrm{~B}$ and $3 \mathrm{~B}$ present the performances for all three tasks for both hands for the children with symptoms of the ADHD subtypes and the non-ADHD comparison group for the age groups $6-9 \mathrm{yr}$ and $10-13 \mathrm{yr}$. As could be expected, the younger group's performance was consistently poorer than the older group on all three tasks. Figure $1 \mathrm{~B}$ shows that it was especially the children with symptoms of ADHD-C and ADHD-HI that encountered the most difficulties on the Grooved Pegboard. This was the case for both age groups for both hands. Figure 2B shows that on the Maze Coordination Task the children with symptoms of the ADHD subtypes' performance were poorer than that of the non-ADHD comparison group. This was true for both the dominant- and the non-dominant hand. The results were more pronounced in the younger group. Figure $3 \mathrm{~B}$ illustrates that in both age groups there was little difference in finger tapping performance between the group with symptoms of the ADHD subtypes and the non-ADHD comparisons.

The effects of ADHD subtype symptoms, gender and age for all three tests are shown in Table 2. There were statistically significant main effects of symptoms of ADHD subtype (ps $<0.001$ ) as well as a significant 3-way interaction effect between hand dominance $\times$ age $\times$ ADHD subtype symptoms $(\mathrm{p}=0.018$ and $\mathrm{p}=0.043$, respectively $)$. On all three tests, there was also a statistically significant effect of age $(\mathrm{p}<0.001)$. 


\section{A. Effect of Gender}

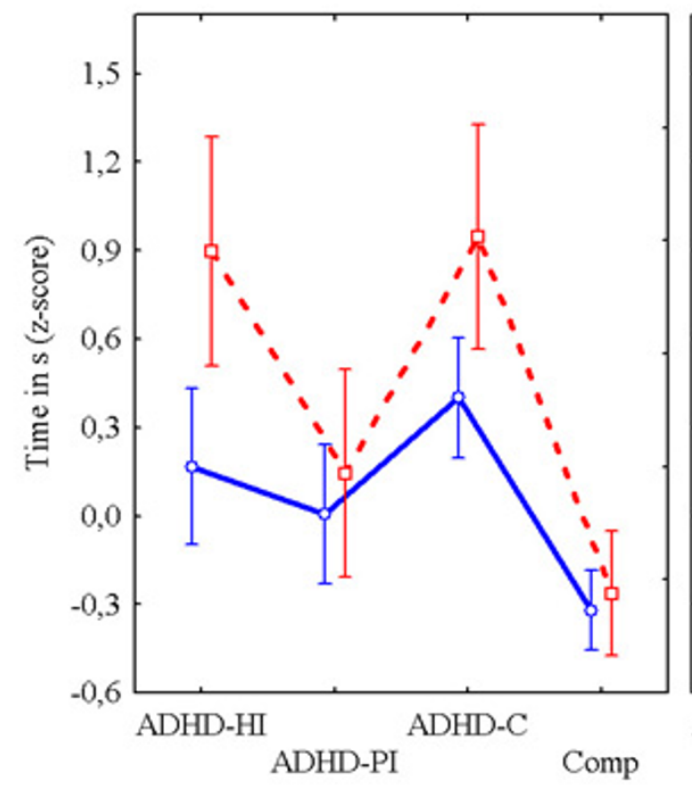

Dominant Hand

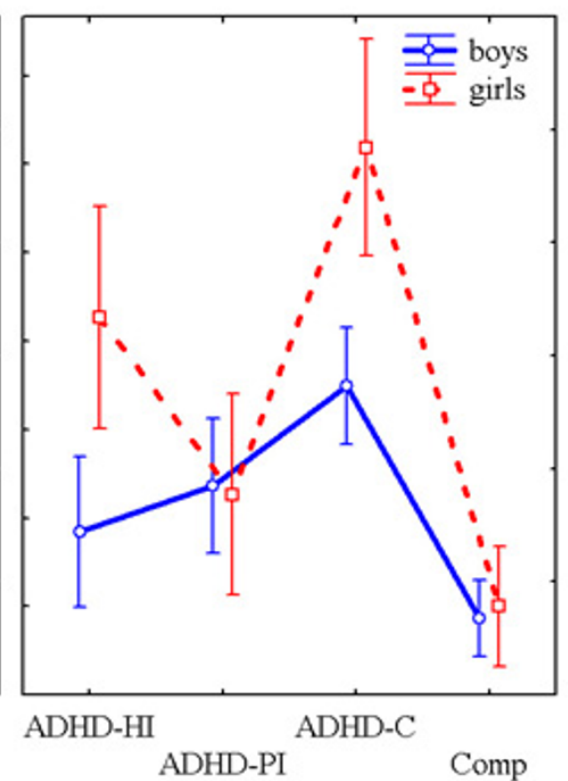

Non-Dominant Hand

\section{B. Effect of Age}

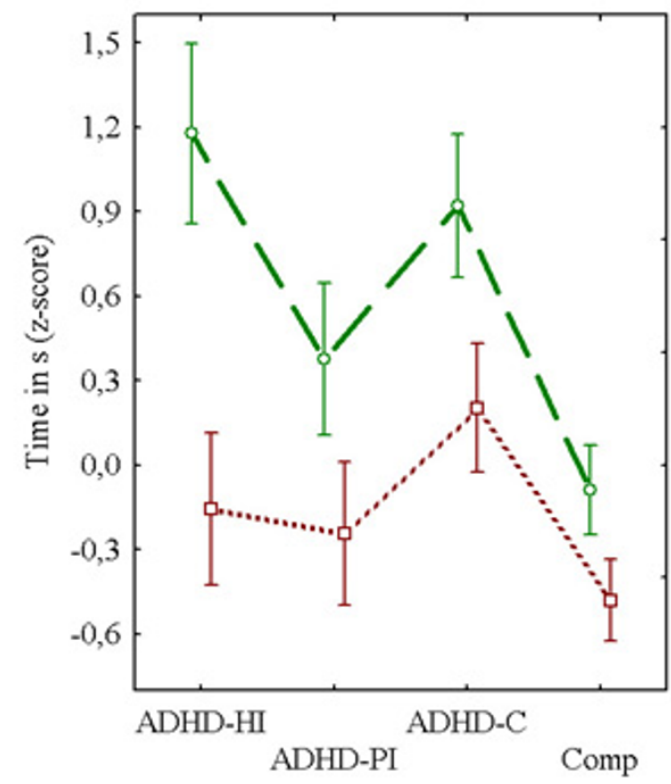

Dominant Hand

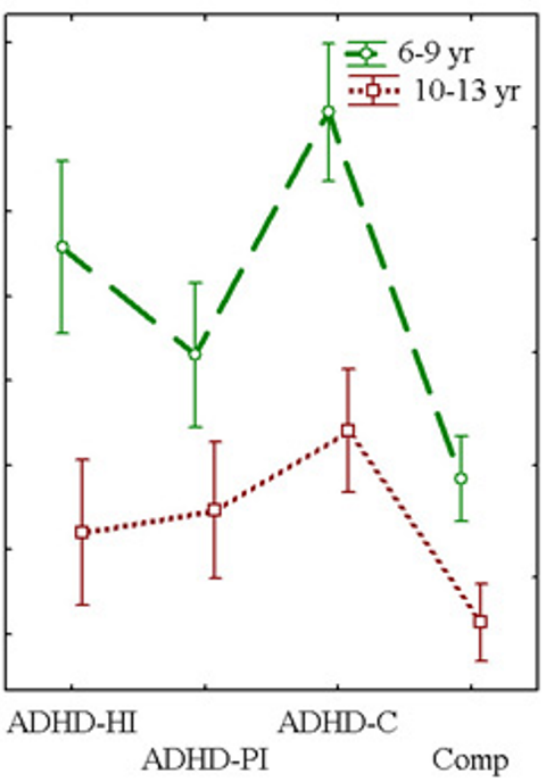

Non-Dominant Hand

\section{Figure I}

Grooved Pegboard. A. The graphs represents the means \pm SEM of the time used by the groups with symptoms of the three ADHD subtypes and the non-ADHD comparison group to complete the grooved Pegboard, as a function of gender. The left side graph illustrates the time used with the dominant hand, while the one on the right side represents the time to complete the task with the non-dominant hand. B. Illustration of the means \pm SEM of the time used by the groups with symptoms of the three ADHD subtypes and the non-ADHD comparison group to complete the Grooved Pegboard task as a function of age. The left side graph shows the performance with the dominant hand, while the right side graph shows the time taken with the non-dominant hand. 


\section{A. Effect of Gender}

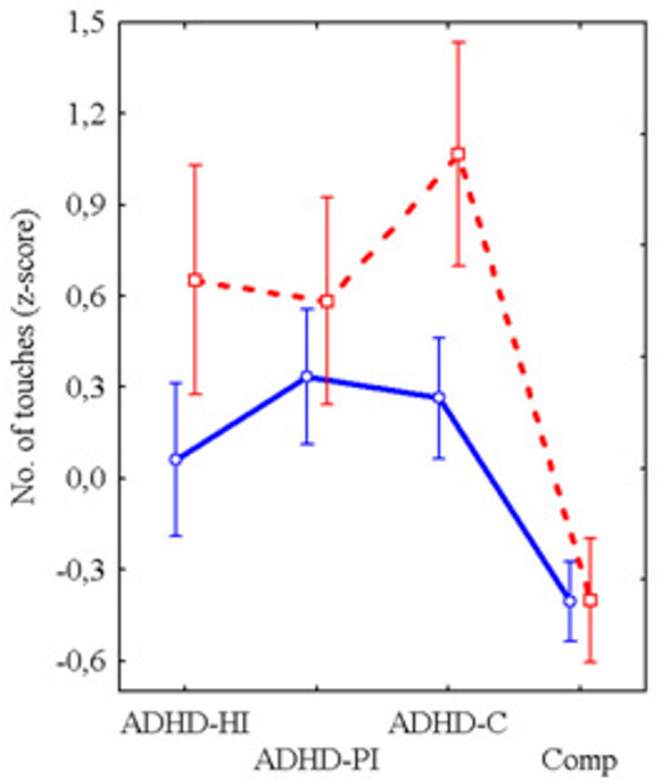

Dominant Hand

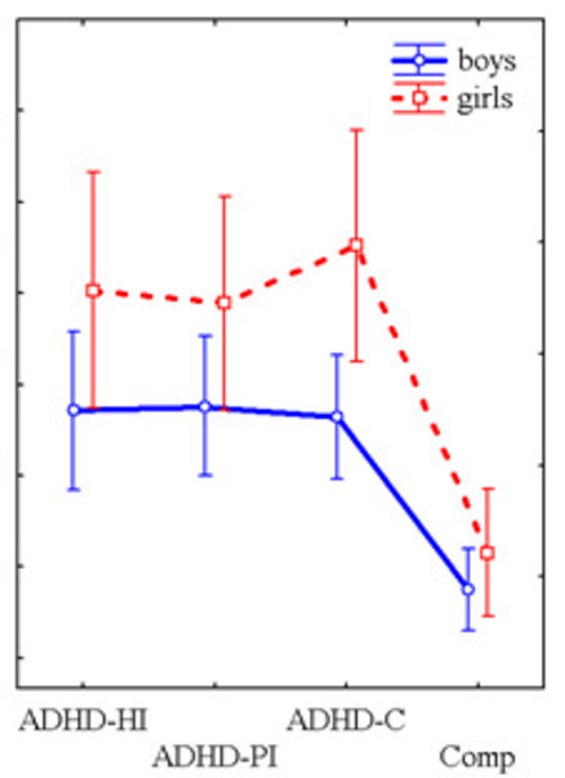

Non-Dominant Hand

\section{B. Effect of Age}

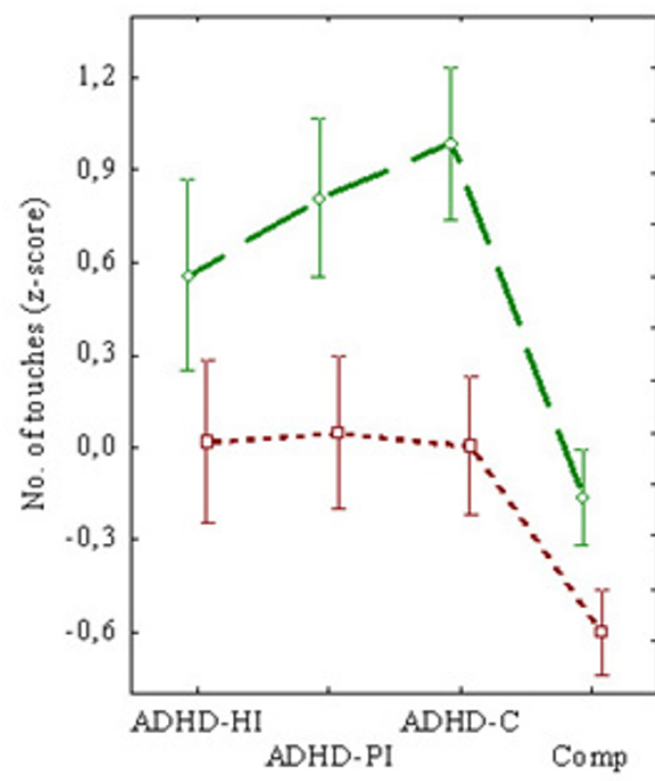

Dominant Hand

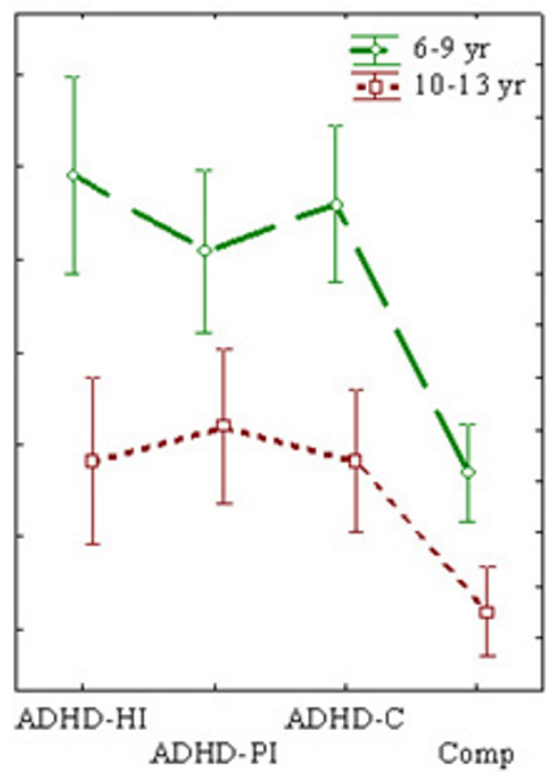

Non-Dominant Hand

\section{Figure 2}

Maze Coordination Task. A. The graph shows the means \pm SEM of number of touches against the sides of the maze made by the groups with symptoms of the three ADHD subtypes and the non-ADHD comparison group as a function of gender. The left side graph shows the performance with the dominant hand, while the right side graph shows the number of touches with the non-dominant hand. $\mathbf{B}$. The graph shows the means \pm SEM of the number of touches against the sides of the maze made by the groups with symptoms of the three ADHD subtypes and the non-ADHD comparison group as a function of age. The left side graph shows the performance with the dominant hand, while the right side graph shows the results with the non-dominant hand. 


\section{A. Effect of Gender}

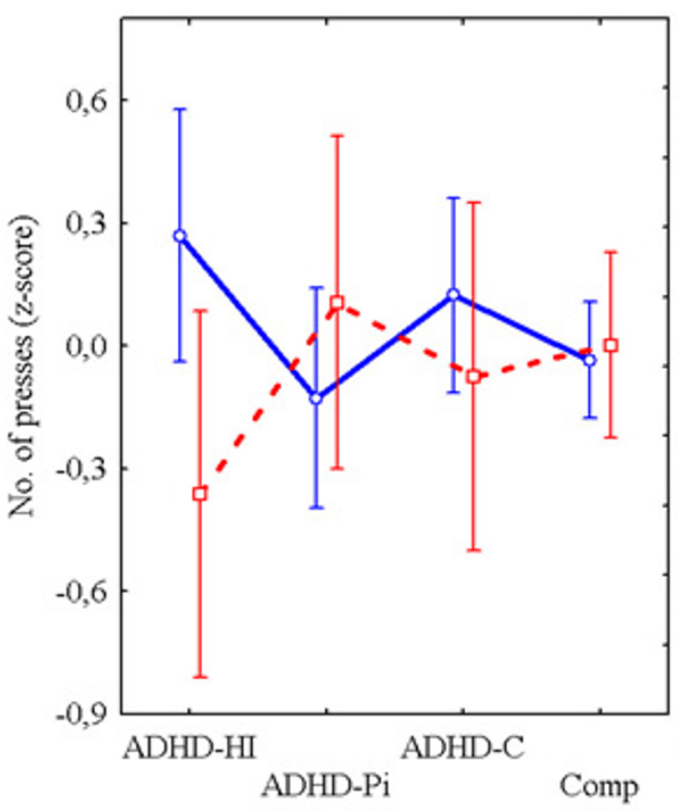

Dominant Hand

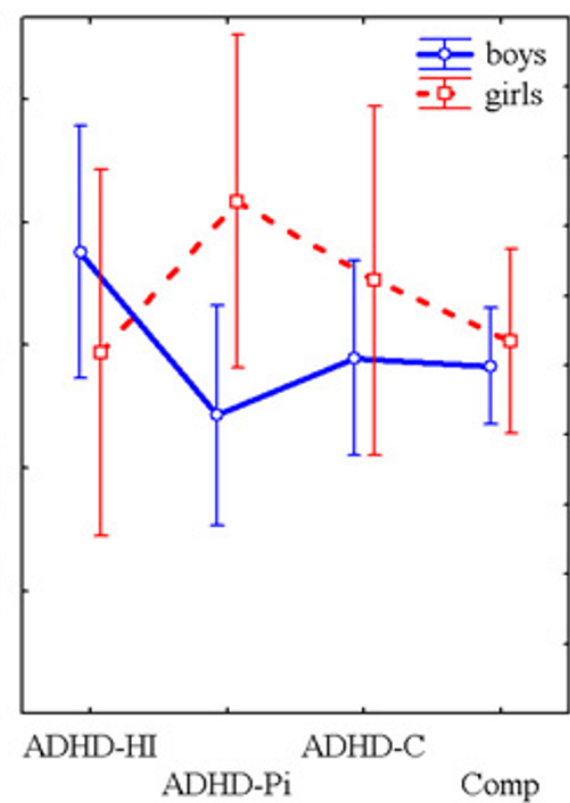

Non-Dominant Hand

B. Effect of Age

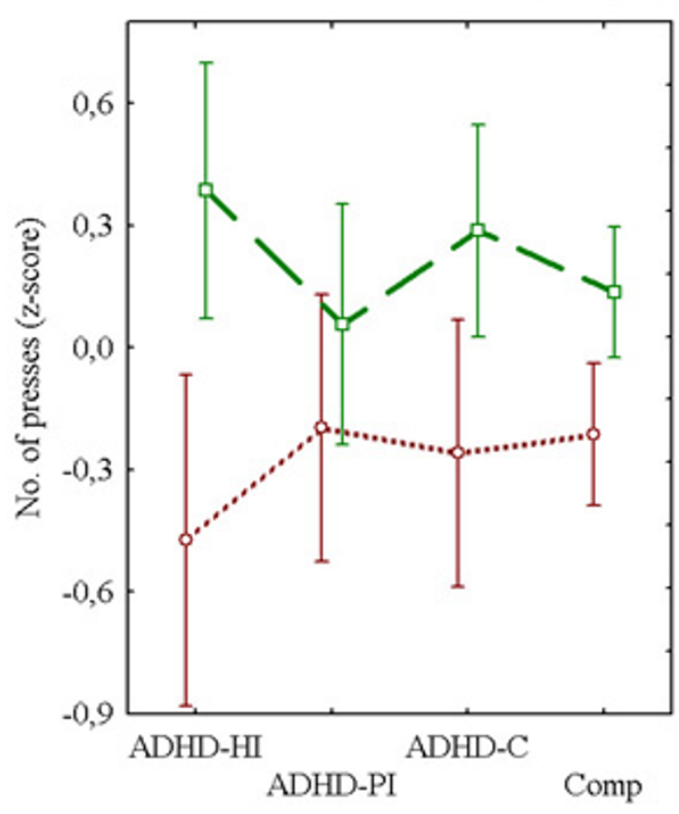

Dominant Hand

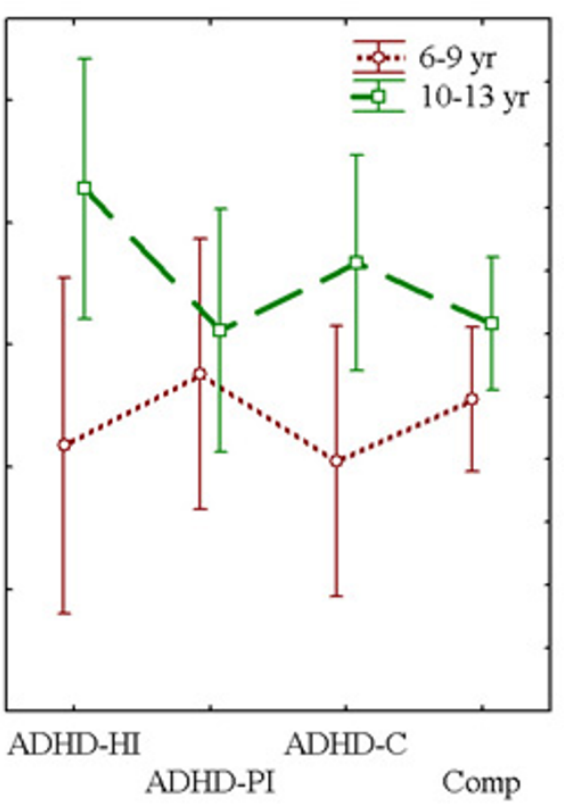

Non-Dominant Hand

Figure 3

Finger Tapping Test. A. The means \pm SEM of the number of presses are illustrated for the groups with symptoms of the three ADHD subtypes and the non-ADHD comparison group. The left hand graph shows the number of presses with the dominant hand while the right hand graph shows the performance with the non-dominant hand as a function of gender. B. The means \pm SEM of the number of presses are depicted for the groups with symptoms of the three ADHD subtypes and the nonADHD comparison group as a function of age. The left side graph shows the performance with the dominant hand and the right side graph with the non-dominant hand. 
Table 2: Results from repeated measures ANOVA

\begin{tabular}{|c|c|c|c|c|}
\hline \multirow[t]{2}{*}{ Measure } & \multirow[t]{2}{*}{ Variable } & \multicolumn{3}{|c|}{ ANOVA } \\
\hline & & & Gender & Age \\
\hline \multirow[t]{8}{*}{ Grooved Pegboard } & & Df & $\mathbf{F}$ & $\mathbf{F}$ \\
\hline & ADHD Subtype & 3,512 & 14. $270^{* * * *}$ & $40.936 * * *$ \\
\hline & Gender/Age & 1,512 & $2895.916 * * *$ & $105.042^{* * *}$ \\
\hline & Gender/Age $\times$ Subtype & 3,512 & $30.504^{* *}$ & $5.216^{* * *}$ \\
\hline & Dominance & 1,512 & 47. $812^{* * * *}$ & 0.308 \\
\hline & Dominance $\times$ Gender/Age & 1,512 & 0.328 & 0.206 \\
\hline & Dom $\times$ Subtype & 3,512 & 4. $790 * *$ & $3.290^{*}$ \\
\hline & Dom $\times$ Gender/Age $\times$ Subtype & 3,512 & 2.285 & $3.373^{*}$ \\
\hline \multicolumn{5}{|l|}{ Maze Coordination } \\
\hline & ADHD Subtype & 3,516 & $26.006 * * *$ & $37.57 \mid * * * *$ \\
\hline & Gender/Age & 1,516 & $13.694^{* * *}$ & $75.464 * * *$ \\
\hline & Gender/Age $\times$ Subtype & 3,516 & 1.672 & 2.244 \\
\hline & Dominance & 1,516 & $198.165^{* * * *}$ & 0.050 \\
\hline & Dominance $\times$ Gender/Age & I, 516 & 0.045 & 0.055 \\
\hline & Dom $\times$ Subtype & 3,516 & 1.134 & $3.122 *$ \\
\hline & Dom $\times$ Gender/Age $\times$ Subtype & 3,516 & 0.414 & $2.729 *$ \\
\hline \multicolumn{5}{|l|}{ Finger Tapping } \\
\hline & ADHD Subtype & 3,475 & 0.437 & 0.069 \\
\hline & Gender/Age & $\mathrm{I}, 475$ & 2.584 & 19.961 **** \\
\hline & Gender/Age $\times$ Subtype & 3,475 & $3.088^{*}$ & 1.596 \\
\hline & Dominance & $\mathrm{I}, 475$ & $61.979 * * *$ & 0.304 \\
\hline & Dominance $\times$ Gender/Age & I, 475 & $8.290 * *$ & 2.581 \\
\hline & Dom $\times$ Subtype & 3,475 & 0.589 & 0.493 \\
\hline & Dom $\times$ Gender $/$ Age $\times$ Subtype & 3,475 & 1.572 & 0.120 \\
\hline
\end{tabular}

$$
\begin{aligned}
& * \mathrm{p}<0.05 \\
& * * \mathrm{p}<0.01 \\
& * * * \mathrm{p}<0.001
\end{aligned}
$$

Bonferroni corrected post-hoc tests on the Grooved Pegboard and the Maze Coordination Task showed that again, that the groups with symptoms of the ADHD-C subtype had the poorest performance. This was however, only in the case in the younger group. No significant effect was found in the difference between the older children with symptoms of ADHD-C and their non-ADHD comparisons. Independent of hand used, the younger children with symptoms of ADHD-HI differed significantly from the comparisons in the Grooved Pegboard, but not in the Maze Coordination Task. Similarly, independent of hand used, the younger children with ADHD-PI symptoms differed significantly from the comparisons on the Maze Coordination Task, but only with the non-dominant hand on the Grooved Pegboard. The older group (10 13) did not differ significantly, from the non-ADHD comparisons in any of the tasks.

\section{Discussion}

The primary aim of the present study was to investigate if there is an association between symptoms of ADHD and motor skills, in African cultures also, as has already been implicated in Western studies $[8,17,24,40,41]$ and whether these motor problems will differ as a function of ADHD subtype, gender, age, and hand dominance. The study compared South African children from seven different ethnic groups with symptoms of the three ADHD subtypes with a Non-ADHD comparison group on three measures of motor functioning: the Grooved Pegboard, which measures manual dexterity, complex coordination and movement speed; the Maze Coordination Task, which measures complex coordination, goal-directed fine movements, accuracy and stability of movement; and the Finger Tapping Test, which is a simple measure of finger movement and speed [42]. The tasks were performed with both the dominant and the non-dominant hands because when a task is performed with the non-dominant hand, it becomes more complex, probably because it requires continuous attention and more control $[43,44]$.

\section{Association of motor problems with ADHD}

The performance of all groups with symptoms of the three ADHD subtypes was significantly poorer on the Grooved Pegboard and the Maze Coordination Task than that of 
the non-ADHD comparisons. Maybe because the Finger Tapping Test is a simple measure of motor speed and does not involve complex coordination and goal-directed movements, there was no significant difference in performance between the children with ADHD symptomatology and the non-ADHD comparison group on this test. This result replicates that of a European study [45]. Deficits in motor control in ADHD have been reported previously especially when more complex motor sequences have to be performed [43]. Barkley [2] and Leung and Connolly [46] ascribe this to dysfunctional higher-order cognitive processes such as planning and behavioural organising, involved in the more complex motor tasks. However, not all researchers share this opinion. According to Sagvolden and co-workers $[15,16]$ the neurobiological basis is predicted to be a hypofunctioning nigro-striatal dopaminergic system. Neuropsychological studies indicate that the areas involved in ADHD includes the basal ganglia, as well as the cerebellum and the prefrontal cortex [47].

The results of the present study show that the groups with ADHD symptoms were less impaired on the speeded task (Grooved Pegboard) than on the more complex Maze Coordination Task which requires more control, stability, and motor planning. The poor performance on the Maze Coordination Task indicated that children with ADHD symptoms appear to have problems with eye-hand coordination, and control of the task by means of prestructured motor plans [18] as this tasks requires planning ahead. The poorer performance on the Grooved Pegboard especially of the children with symptoms of ADHD-C, suggested that their eye-hand coordination is impaired when motor speed is required $[48,49]$.

\section{Subtypes}

All three subgroups showing symptoms of the ADHD had motor performance problems when compared to children without ADHD symptoms. The group with ADHD-C symptoms performed significantly poorer on both the Grooved Pegboard and the Maze Coordination Task, while there were no significant differences for the Finger Tapping Test. It is interesting to notice that the group with ADHD-PI symptoms only differed significantly from the comparison group on the Maze Coordination Task, but not on the Grooved Pegboard. An explanation may be that the Maze Coordination Task is slightly more complex than the Grooved Pegboard, which measures accuracy, and motor speed, but not the same degree of complex eyehand coordination and motor planning as is required by the Maze Coordination Task.

Only the girls with ADHD-HI symptoms differed significantly from the comparison group in both the Grooved Pegboard and Maze Coordination Task. This was however only the case with the dominant hand. In general, the findings were in line with other studies which found the most pronounced impairment in the children with symptoms of ADHD-C and ADHD-PI subtypes [23,24,41,5052]. An association between symptoms of inattention and poor motor skills is well-documented [17,23,41]. The study by Pitcher, Piek and Hay [41] found that $58 \%$ of children with ADHD-PI, $49 \%$ of ADHD-C, and $47 \%$ of ADHD-HI were having motor problems. The present study also supports the findings of Hinshaw and co-workers, using the Grooved Pegboard in girls with ADHD, that most impairments are found in the ADHD-C subtype with the ADHD-PI group impaired to a lesser degree [53]. This finding is remarkable as the scores on the Inattention scale of the DBD rating scale did not differ between the two groups $(22.86 \pm 2.65$ vs. $22.84 \pm 3.07)$ and a strong link between inattentiveness and motor dyscontrol has been reported in most studies $[22,23,41]$. A possible explanation may be that the additional hyperactivity/impulsiveness symptoms add to the impairment of children with symptoms of ADHD-C. The reason may be that poor fine motor skills make greater demands on sustained attention; therefore fine motor movements will be more affected in children with attention deficits than comparison children performing fine motor skills smoothly [41]. There is a strong association between inattention and movement difficulties, as a more pronounced inattention predicts more difficulties in motor coordination [23,41]. The lesser association of ADHD-HI symptoms with motor problems is also confirmed by most studies [41]. Impulsiveness has been associated with motor problems by Tseng and co-workers [54], their explanation was that impulsive children are more inaccurate and do not learn from their mistakes.

\section{Gender differences}

Sex differences have only been infrequently assessed in the literature. Gaub and Carlson in their meta-analysis [55] found no difference in motor skills between the genders. In the present study, girls performed both the Grooved Pegboard and the Maze Coordination Task significantly poorer than the boys. This was however only the case with the dominant hand. This finding may support the statement by Biederman and co-workers [56] that, although ADHD is less frequent in girls, the symptoms are more severe than in boys.

\section{Age effect}

Age was the most pronounced of the statistical effects. For all measures, except one, significant differences were only found in the younger group with symptoms of ADHD, when compared with the non-ADHD comparison group. The exceptions were the children with ADHD-C symptoms on the Grooved Pegboard. Independent of hand used, their performance was significantly poorer than that 
of the comparison group without ADHD symptoms. Some studies show that, although some children seem to outgrow their motor problems, they often persist into adulthood [57]. The results could be attributed to the effect of maturation on neuropsychological performance [21] and therefore the tasks could have been insensitive to differences in motor functioning between older children with and without symptoms of ADHD in the present study.

\section{Hand dominance}

When the children with symptoms of ADHD showed significantly poorer performance with one hand only, it was the dominant hand. This was the case for both the boys and girls with ADHD-PI symptoms in the Maze Coordination Task and for only the girls with ADHD-HI symptoms on the Grooved Pegboard. This supports the findings of Kalff and co-workers [43] that children at risk for ADHD were disproportionately more inaccurate and had more unstable performance with their preferred hand than other children. The exception was the result of the younger boys with symptoms of ADHD-HI on the Maze Coordination Task where there was a significant poorer performance with the non-dominant hand when compared with their non-ADHD comparisons.

\section{Conclusion}

This study shows that African children from different ethic groups with ADHD symptoms have poorer motor control, accuracy and speed when the tasks are fairly complex like in the Grooved Pegboard and Maze coordination Task. There was no observable difference when the task consists of a simple motor movement, like in the Finger Tapping Test. This deficiency in motor functioning is found in all ADHD subtypes, with the group with symptoms of ADHD-C most severely affected.

The study did generally not reveal striking gender differences; the exception was the significantly poorer performance of the ADHD-HI girls with the dominant hand on both the Grooved Pegboard and the Maze Coordination Task. The boys did not differ significantly from their nonADHD comparisons when using the dominant hand. This result is difficult to explain, as it was only found in one ADHD subtype, but it cannot be attributed to chance, as it was observed in both the Grooved Pegboard and the Maze Coordination Task.

The results also showed that problems with motor control were less noticeable in the older groups, probably due to the effect of maturation, which made the tasks too easy for this particular age group.

Table 3: Comparing ADHD subtypes with non-ADHD groups

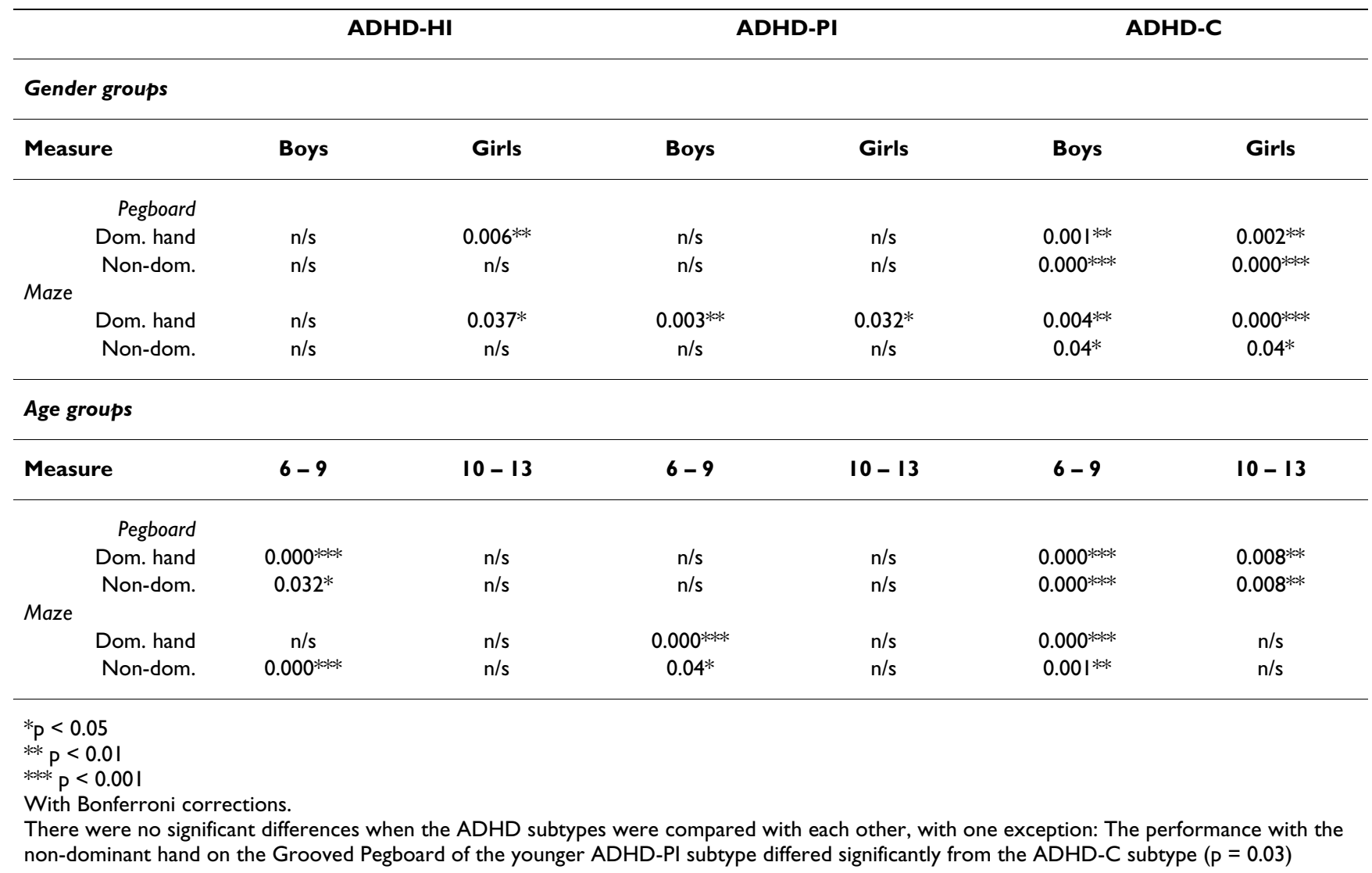


Usually there were no differences between performances with the dominant and non-dominant hand when the children with ADHD symptoms were compared with the non-ADHD comparison group, the exception being the girls with ADHD-HI symptoms who performed significantly poorer with their dominant hand on two of the tasks than the non-ADHD girls. The younger boys on the other hand did have problems only on the Maze Coordination Task with their non-dominant hand.

The present study shows that problems with motor control in children with ADHD symptoms are not associated with culture or ethnicity, were present in all three ADHD subtypes, that both genders are affected, are less pronounced in older children, and that the problems exist in both hands. These are significant findings as children with motor problems are at risk for learning problems and poorer psychological adjustment $[50,58]$. Because motor clumsiness is not a diagnostic criteria in DSM-IV [1], it is often not assessed when ADHD is diagnosed and the child may go without intervention.

\section{Abbreviations}

ADHD - Attention-Deficit/hyperactivity Disorder

ADHD-C - ADHD (Combined subtype)

ADHD-HI - ADHD (Hyperactive/impulsive subtype)

ADHD-PI - ADHD (Predominantly Inattentive subtype)

DBD - Disruptive Behavior Disorders rating scale

\section{Competing interests}

The author(s) declare that they have no competing interests.

\section{Authors' contributions}

AM participated in the development of the study design, supervised the data collection, prepared the data, performed the statistical analysis, and wrote the manuscript.

TS participated in the development of the study design, worked on the manuscript and approved the final draft.

\section{Acknowledgements}

This study was funded by the Norwegian Universities' Committee for Development Research and Education (NUFU) Psychology Cooperation Programme between the University of Oslo and the University of Limpopo. The authors gratefully acknowledge Eunice Mashego, Mudzunga Mathivha, Ruth Chuene, Tshikani Nkanyani, Gloria Pila, Estelle McAlpine, and Jan Lekalakala for help in data collection and administration.

\section{References}

I. American Psychiatric Association: Diagnostic and statistical manual of mental disorders: DSM-IV-TR Washington DC, Author; 2000.
2. Barkley RA: Attention-deficit hyperactivity disorder: A handbook for diagnosis and treatment. 2nd edition. New York, The Guilford Press; 1998.

3. Swanson JM, Sergeant JA, Taylor E, Sonuga-Barke EJS, Jensen PS, Cantwell DP: Attention-deficit hyperactivity disorder and hyperkinetic disorder. Lancet 1998, 35 1:429-433.

4. Taylor E: Clinical foundations of hyperactivity research. Behav Brain Res 1998, 94: | |-24.

5. Biederman J, Faraone SV, Spencer T, Wilens T, Mick E, Lapey KA: Gender differences in a sample of adults with attention deficit hyperactivity disorder. Psychiatry Res 1994, 53:13-29.

6. American Academy of Pediatrics: Clinical practice guideline: diagnosis and evaluation of the child with attention-deficit/ hyperactivity disorder. Pediatrics 2000, I 05: I I 58-I I 70.

7. Faraone SV, Biederman J, Weber W, Russell RL: Psychiatric, neuropsychological, and psychosocial features of DSM-IV subtypes of attention-deficit/hyperactivity disorder: results from a clinically referred sample. J Am Acad Child Adolesc Psychiatry 1998, 37:185-193.

8. Kadesjo B, Gillberg C: The comorbidity of ADHD in the general population of Swedish school-age children. J Child Psychol Psychiatry 2001, 42:487-492.

9. Newcorn JH, Halperin JM, Jensen PS, Abikoff HB, Arnold LE, Cantwell DP, Conners CK, Elliott GR, Epstein JN, Greenhill LL, Hechtman L, Hinshaw SP, Hoza B, Kraemer HC, Pelham WE, Severe JB, Swanson JM, Wells KC, Wigal T, Vitiello B: Symptom profiles in children with ADHD: effects of comorbidity and gender. J Am Acad Child Adolesc Psychiatry 200 I, 40: I37-I46.

10. Biederman J, Wilens T, Mick E, Spencer T, Faraone SV: Pharmacotherapy of attention-deficit/hyperactivity disorder reduces risk for substance use disorder. Pediatrics 1999, 104:e20.

II. Meyer A, Aase H: Assessment and intervention in childhood disruptive behaviour disorders. In Contributions to psychotherapy in Africa. Edited by: Madu NS. Polokwane (Pietersburg), UNIN Press; 2003:164-178.

12. Alarcon RD, Westermeyer J, Foulks EF, Ruiz P: Clinical relevance of contemporary cultural psychiatry. J Nerv Ment Dis 1999 , I 87:465-47|.

13. Meyer A: Attention Deficit/Hyperactivity Disorder among North Sotho speaking primary school children in South Africa: Prevalence and sex ratios. I Psychol in Afr 1998, 8:186-195.

14. Meyer A, Eilertsen DE, Sundet JM, Tshifularo JG, Sagvolden T: Crosscultural similarities in ADHD-like behaviour amongst South African primary school children. S Afr J Psychol 2004, 34:123-139.

15. Johansen EB, Aase H, Meyer A, Sagvolden T: Attention-Deficit/ Hyperactivity Disorder (ADHD) behaviour explained by dysfunctioning reinforcement and extinction processes. Behav Brain Res 2002, I 30:37-45.

16. Sagvolden T, Johansen EB, Aase H, Russell VA: A dynamic developmental theory of attention-deficit/hyperactivity disorder (ADHD) predominantly hyperactive/impulsive and combined subtypes. Behav Brain Sci 2005, 28:397-4I9.

17. Piek JP, Dyck MJ: Sensory-motor deficits in children with developmental coordination disorder, attention deficit hyperactivity disorder and autistic disorder. Hum Mov Sci 2004, 23:475-488

18. Schoemaker MM, Ketelaars CE, van ZM, Minderaa RB, Mulder T: Deficits in motor control processes involved in production of graphic movements of children with attention-deficit-hyperactivity disorder. Dev Med Child Neurol 2005, 47:390-395.

19. Jongmans MJ, Smits-Engelsman BC, Schoemaker MM: Consequences of comorbidity of developmental coordination disorders and learning disabilities for severity and pattern of perceptual-motor dysfunction. J Learn Disabil 2003, 36:528-537.

20. Flapper BC, Houwen S, Schoemaker MM: Fine motor skills and effects of methylphenidate in children with attention-deficithyperactivity disorder and developmental coordination disorder. Dev Med Child Neurol 2006, 48:165-169.

21. Sergeant JA, Piek JP, Oosterlaan J: ADHD and DCD: A relationship in need of research. Hum Mov Sci 2006, 25:76-89.

22. Martin NC, Piek JP, Hay D: DCD and ADHD: A genetic study of their shared aetiology. Hum Mov Sci 2006, 25: I I0-I 24.

23. Piek JP, Pitcher TM, Hay DA: Motor coordination and kinaesthesis in boys with attention deficit- hyperactivity disorder. Dev Med Child Neurol 1999, 4I: I59-165. 
24. Gillberg C: Deficits in attention, motor control, and perception: a brief review. Arch Dis Child 2003, 88:904-910.

25. Pereira HS, Landgren N, Gillberg C, Forssberg H: Parametric control of fingertip forces during precision grip lifts in children with DCD (developmental coordination disorder) and DAMP (deficits in attention motor control and perception). Neuropsychologia 200I, 39:478-488.

26. Peters JM, Barnett AL, Henderson SE: Clumsiness, dyspraxia and developmental co-ordination disorder: how do health and educational professionals in the UK define the terms? Child Care Health Dev 2001, 27:399-4I2.

27. Purvis $\mathrm{KL}$, Tannock $\mathrm{R}$ : Language abilities in children with attention deficit hyperactivity disorder, reading disabilities, and normal controls. J Abnorm Child Psychol 1997, 25: I33-। 44.

28. Kooistra L, Crawford S, Dewey D, Cantell M, Kaplan BJ: Motor correlates of ADHD: contribution of reading disability and oppositional defiant disorder. J Learn Disabil 2005, 38: 195-206.

29. Ghaziuddin M, Tsai LY, Ghaziuddin N: Brief report: a reappraisal of clumsiness as a diagnostic feature of Asperger syndrome. J Autism Dev Disord 1992, 22:65 I-656.

30. Gillberg C: Asperger syndrome in $\mathbf{2 3}$ Swedish children. Dev Med Child Neurol 1989, 31:520-531.

31. Pelham WEJ, Gnagy EM, Greenslade KE, Milich R: Teacher ratings of DSM-III-R symptoms for the disruptive behavior disorders. J Am Acad Child Adolesc Psychiatry 1992, 31:210-2 18.

32. Pillow DR, Pelham WEJ, Hoza B, Molina BS, Stultz CH: Confirmatory factor analyses examining attention deficit hyperactivity disorder symptoms and other childhood disruptive behaviors. J Abnorm Child Psychol 1998, 26:293-309.

33. Matthews C], Kløve H: Instruction manual for the Adult Neuropsychology Test Battery Madison, University of Wisconsin Medical School; 1964.

34. Reitan RM, Wolfson D: The Halstead-Reitan Neuropsychological Test Battery Tuscon, AZ, Neuropsychological Press; 1985.

35. Van Eeden R: Manual for the South African Individual Scale - Revised (SSAIS-R) Pretoria, Human Sciences Research Council; 1997.

36. Raven J: The Raven's progressive matrices: change and stability over culture and time. Cognit Psychol 2000, 4 I: I-48.

37. Wilkes ], Weigel A: [Comparison of WISC-R and Raven's Progressive Matrices tests in a clinical consultation population]. Z Kinder Jugendpsychiatr Psychother 1998, 26:26I-265.

38. Hodges JR: Cognitive assessment for clinicians New York, Oxford University Press; 1994.

39. StatSoft: STATISTICA for Windows. 2003 [http:// www.statsoft.com]. Tulsa, OK: StatSoft, Inc.

40. Blondis TA: Motor disorders and attention-deficit/hyperactivity disorder. Pediatr Clin North Am 1999, 46:899-9/3.

41. Pitcher TM, Piek JP, Hay DA: Fine and gross motor ability in males with ADHD. Dev Med Child Neurol 2003, 45:525-535.

42. Lezak MD, Howieson DB, Loring DW: Neuropsychological Assessment 4th ed edition. New York, Oxford University Press; 2004.

43. Kalff AC, de Sonneville LM, Hurks PP, Hendriksen JG, Kroes M, Feron FJ, Steyaert J, van Zeben TM, Vles JS, Jolles J: Low- and high-level controlled processing in executive motor control tasks in 5 6-year-old children at risk of ADHD. J Child Psychol Psychiatry 2003, 44: 1049-1057.

44. Pryde KM, Bryden PJ, Roy EA: A developmental analysis of the relationship between hand preference and performance: $I$. Preferential reaching into hemispace. Brain Cogn 2000, 43:370-374.

45. Seidman LJ, Biederman J, Faraone SV, Weber W, Ouellette C: Toward defining a neuropsychology of attention deficithyperactivity disorder: performance of children and adolescents from a large clinically referred sample. J Consult Clin Psychol 1997, 65:150-160.

46. Leung PWL, Connolly KJ: Do hyperactive children have motor organization and/or execution deficits? Dev Med Child Neurol 1998, 40:600-607.

47. Castellanos FX, Tannock R: Neuroscience of attention-deficit/ hyperactivity disorder: the search for endophenotypes. Nat Rev Neurosci 2002, 3:617-628.

48. Carte ET, Nigg JT, Hinshaw SP: Neuropsychological functioning, motor speed, and language processing in boys with and without ADHD. J Abnorm Child Psychol 1996, 24:48I-498.

49. Steger J, Imhof K, Coutts E, Gundelfinger R, Steinhausen HC, Brandeis D: Attentional and neuromotor deficits in ADHD. Dev Med Child Neurol 200I, 43: I72-179.
50. Hellgren L, Gillberg IC, Bagenholm A, Gillberg C: Children with deficits in attention, motor control and perception (DAMP) almost grown up: psychiatric and personality disorders at age 16 years. IChild Psychol Psychiatry 1994, 35: I255-I27I.

51. Kadesjo B, Gillberg C: Attention deficits and clumsiness in Swedish 7-year-old children. Dev Med Child Neurol 1998, 40:796-804

52. Dewey D, Kaplan BJ, Crawford SG, Wilson BN: Developmental coordination disorder: associated problems in attention, learning, and psychosocial adjustment. Hum Mov Sci 2002, 21:905-9|8.

53. Hinshaw SP, Carte ET, Sami N, Treuting J], Zupan BA: Preadolescent girls with attention-deficit/hyperactivity disorder: II. Neuropsychological performance in relation to subtypes and individual classification. J Consult Clin Psychol 2002, 70: I099-IIII.

54. Tseng MH, Henderson A, Chow SM, Yao G: Relationship between motor proficiency, attention, impulse, and activity in children with ADHD. Dev Med Child Neurol 2004, 46:38I-388.

55. Gaub M, Carlson CL: Gender differences in ADHD: a metaanalysis and critical review. I Am Acad Child Adolesc Psychiatry 1997, 36: 1036-1045.

56. Biederman J, Faraone SV, Mick E, Williamson S, Wilens TE, Spencer T], Weber W, Jetton J, Kraus I, Pert J, Zallen B: Clinical correlates of ADHD in females: findings from a large group of girls ascertained from pediatric and psychiatric referral sources. J Am Acad Child Adolesc Psychiatry 1999, 38:966-975.

57. Visser J: Developmental coordination disorder: a review of research on subtypes and comorbidities. Hum Mov Sci 2003, 22:479-493.

58. Landgren M, Kjellman B, Gillberg C: "A school for all kinds of minds." The impact of neuropsychiatric disorders, gender and ethnicity on school-related tasks administered to 9-10year-old children. Eur Child Adolesc Psychiatry 2003, I2:162-171.

Publish with Bio Med Central and every scientist can read your work free of charge

"BioMed Central will be the most significant development for disseminating the results of biomedical research in our lifetime. "

Sir Paul Nurse, Cancer Research UK

Your research papers will be:

- available free of charge to the entire biomedical community

- peer reviewed and published immediately upon acceptance

- cited in PubMed and archived on PubMed Central

- yours - you keep the copyright
BiolMedcentral 